\title{
Myelofibrose
}

\section{JAK-Inhibitor Ruxolitinib als zukünftige neue Behandlungsoption?}

\begin{abstract}
Wie die Ergebnisse der Phase-III-Studien COMFORT (Controlled Myelofibrosis Study with Oral JAK Inhibitor Treatment) zeigen, könnte Ruxolitinib eine wichtige neue Behandlungsoption für Patienten mit Myelofibrose werden, so Dr. Srdan Verstovsek, Houston/USA. In den Studien konnte eine deutliche Reduktion der Splenomegalie und der krankheitsbedingten Symptome im Vergleich zu Placebo bzw. bester verfügbarer Therapie gezeigt werden (Verstovsek S et al., 2011, J Clin Oncol 29 [Suppl]:\#6500; Harrison CN et al., 2011, JClin Oncol 29 [Suppl]: LBA6501).
\end{abstract}

Charakteristisch für die drei häufigsten Philadelphia-Chromosom-negativen myeloproliferativen Neoplasien - die Myelofibrose (MF), die Polyzythämia vera (PV) und die essenzielle Thrombozythämie (ET) sind Störungen des JAK (Januskinase)-STAT (Signaltransduktoren und Aktivatoren der Transkription)-Signalwegs durch verschiedene Mechanismen, wie z.B. aktivierende Mutationen (Vannucchi AM et al., 2008, Haematologica 93: 972-976). Diese Störungen führen zu einer Hyperaktivierung des für die Hämatopoese, aber auch die Immunfunktion wichtigen Signalwegs, mit in der Folge vermehrter Zellproliferation, Zelldifferenzierung und Zytokinproduktion. Ruxolitinib (INC424) inhibiert JAK1 und JAK2 und kann so regulierend auf die gestörte Signaltransduktion wirken.

\section{COMFORT-I und COMFORT-II: Reduktion des Milzvolumens}

In der doppelblinden Studie COMFORT-I erhielten Patienten mit primärer MF, POST-PV-MF oder Post-ET-MF und Intermediär-2- oder Hochrisiko randomisiert Ruxolitinib (15mg oder $20 \mathrm{mg}$ p.o. zweimal täglich; $n=155$ ) oder Placebo ( $n=151$ ). Die Therapie mit Ruxolitinib führte laut Verstovsek zu einem deutlichen und nachhaltigen klinischen Nutzen: In Woche 24 war der Anteil der Patienten, die eine Reduktion des Milzvolumens um $\geq 35 \%$ erreichten, mit $41,9 \%$ signifikant größer als unter Placebo mit 0,7\% ( $p<0,0001$; primärer End-
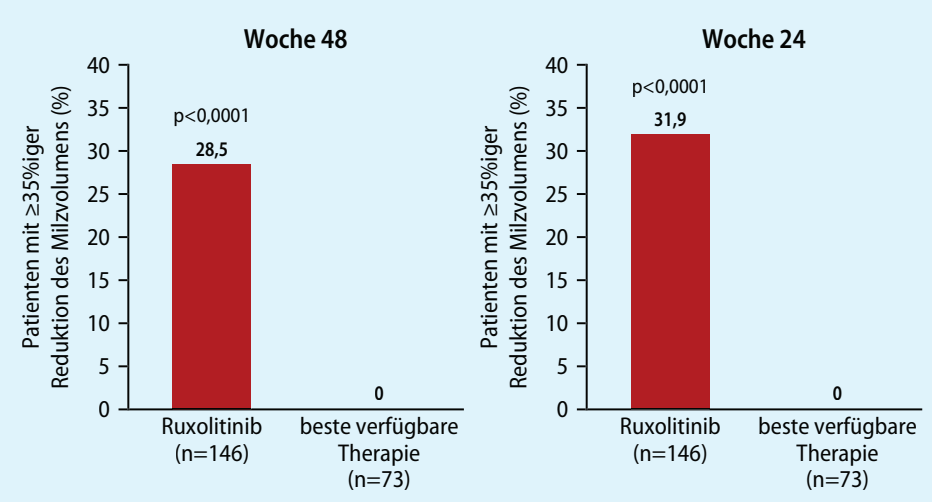

nach Harrison CNet al, 2011 , J Clin Oncol 29 (Suppl): LBA6501

COMFORT-II-Studie: Reduktion des Milzvolumens in Woche 48 (primärer Endpunkt) und Woche 24 (wichtigster sekundärer Endpunkt)

punkt).Zudem konnten Symptome unter Ruxolitinib im Vergleich zu Placebo signifikant besser gelindert werden $(p<0,0001)$. Der Nutzen war unabhängig vom JAK2-Mutationsstatus. Die Therapie wurde insgesamt gut vertragen; zu den häufigsten unerwünschten Wirkungen gehörten Thrombozytopenie und Anämie. Verstovsek betonte, dass diese unerwünschten Wirkungen sowie der Transfusionsbedarfjedoch im Studienverlauf bis nahezu auf Placebo-Niveau zurückgingen.

Die Daten der offen randomisierten Studie COMFORT-II zum Vergleich von Ruxolitinib mit bester verfügbarer Therapie bei einem ähnlichen Patientenkollektiv ergänzen laut Dr. Claire N. Harrison, London/ Großbritannien, die positiven Ergebnisse der COMFORT-I-Studie: Im primären Endpunkt, der Reduktion des Milzvolumens um $\geq 35 \%$ nach 48 Wochen, und im wichtigsten sekundären Endpunkt, der Reduktion des Milzvolumens um $\geq 35 \%$ nach 24 Wochen, zeigte sich ein signifikanter Unterschied zugunsten von Ruxolitinib (Abb.). AM

\section{Metastasierte Weichteil- oder Knochensarkome \\ Erhaltungstherapie mit Ridaforolimus}

Die Behandlung mit dem mTOR-Inhibitor Ridaforolimus kann bei Patienten mit metastasierten Weichteil- oder Knochensarkomen die Krankheitskontrolle im Vergleich zu Placebo verbessern und den Nutzen einer vorangegangenen Chemotherapie länger aufrecht erhalten, schlussfolgerte Dr. Sant P. Chawla, Santa Monica/USA, aus den Ergebnissen der Phase-III-Studie SUCCEED (Chawla SP et al., 2011, J Clin Oncol 29 [Suppl]: \#10005).

711 Patienten, die unter einer vorangegangenen Chemotherapie mindestens eine Krankheitsstabilisierung erreicht hatten, erhielten in dieser Studie randomisiert und doppelblind eine Erhaltungstherapie mit Ridaforolimus (40mg p.o. für fünf Tage pro Woche) oder Placebo bis zur Progression. Eingeschlossen werden konnten Patienten mit metastasierten Weichteil- oder Knochensarkomen, die zuvor mit ein bis drei Chemotherapien behandelt worden waren.

Im primären Endpunkt zeigte sich ein signifikanter Vorteil für die Erhaltungstherapie mit Ridaforolimus mit median 17,7 Wochen pro- gressionsfreiem Überleben versus median 14,6 Wochen progressionsfreies Überleben unter Placebo (Hazard-Ratio 0,72; $p=0,0001$ ). Auch beim klinischen Nutzen (Gesamtansprechrate plus Krankheitsstabilisierung) zeigte sich der Vorteil zugunsten von Ridaforolimus (40,6\% versus $28,6 \% ; p=0,0009$ ). Dabei wurde laut Chawla kein ungünstiger Effekt auf das Überleben der Patienten nach der Krankheitsprogression beobachtet. Bezüglich der unerwünschten Wirkungen ergaben sich keine neuen Aspekte zu den bekannten Nebenwirkungen von mTOR-Inhibitoren. $A M$ 